\title{
REMOTE SYSTEM OF DESIGN AND TEST OF DC-DC CONVERTERS
}

\author{
L. Eguizabal, A Lago, C. Peñalver, J. Doval, J. Marcos and A. Nogueira \\ Electronic Technology Department, University of Vigo
}

\begin{abstract}
The increasing interest in remote test and experimentation has allowed the incorporation to the educative system of new tools that improve the classic methods of education. In the field of the Power Electronics, the possibility of access of the engineering student through the development of this type of tools to equipment generally of high cost, and therefore of low availability in the educational laboratories, is very important. This work shows a Remote System of Design and Test of DC/DC Converters (RSDTC) that can be used either locally or remotely. The developed system is based on a test bench manufactured by Hewlett-Packard. Taking as its starting point this equipment, a software application has been developed using the visual programming language "LabVIEW". This programming tool combined with CGI (Common Gateway Interface), allows the developed analysis system to be executed remotely using a web browser.
\end{abstract}

Key words: Automatic Test Software, DC-DC power conversion, Internet, Power system monitoring, LabVIEW programming.

\section{INTRODUCTION}

Nowadays, with the generalization of the use of PCs in education, new tools have appeared that are very helpful in improving the classic methods of education.

Among these new education tools developed around the use of PCs, it is possible to emphasize:

1. The use of simulation software, which allows multiple kinds of analysis in the study of circuits, thus obtaining results that in any other way would be very difficult to get. 
2. The possibility of improving the presentation methods, as much the theoretical classes as the practical ones.

3. New learning techniques of circuits in the practical classes, based on the connection of programmable instrumentation equipment to a PC, by means of an instrumentation bus.

4. The use of Internet, to facilitate the access to the information available in the network, and also giving the possibility of making practical exercises at distance, which has come to be known as "Remote Experimentation".

This article present a System of Design and Test of DC/DC Converters (RSDTC), that tries to explore the possibilities shown in 3 y 4.

The RSDTC allows the study of this type of power converters in two ways:

a) Design. In this first step the students can use the developed system to determine the values of the passive components that form the circuit output filter of the converter.

b) Test. Once designed the topology, in this phase the quality of the converter is studied. To do this a series of tests have been defined: line regulation, load regulation, etc.

References [1-4] analyzed the main topics of this work.

\section{THE TEST BENCH OF DC-DC CONVERTERS}

The electronic hardware necessary to implement the test system is based on a set of programmable instruments interconnected using the GPIB bus. In order to be able to control and supervise these instruments, a PC is used. The PC sits in one of its PCI expansion buses a GPIB card of the manufacturer National Instruments. The main instruments that compound the test bench are shown in figure 1. It is interesting to emphasize the configuration and interconnection elements.

In order to interconnect the Unit Under Test (UUT) and the instruments, the following element has been used: HP75000. This instrument works like controller of the test bench. In addition it has connected in its VXI expansion slots: three $4^{*} 4$ matrixes of contacts HPE136A cards, and 2 HPE1664 cards of 16 relays.

The relays are used for interconnecting the instruments and the UUT, and for modifying the operation mode of the topology; for example, changing between several inductances and capacitors connected to the converter. 


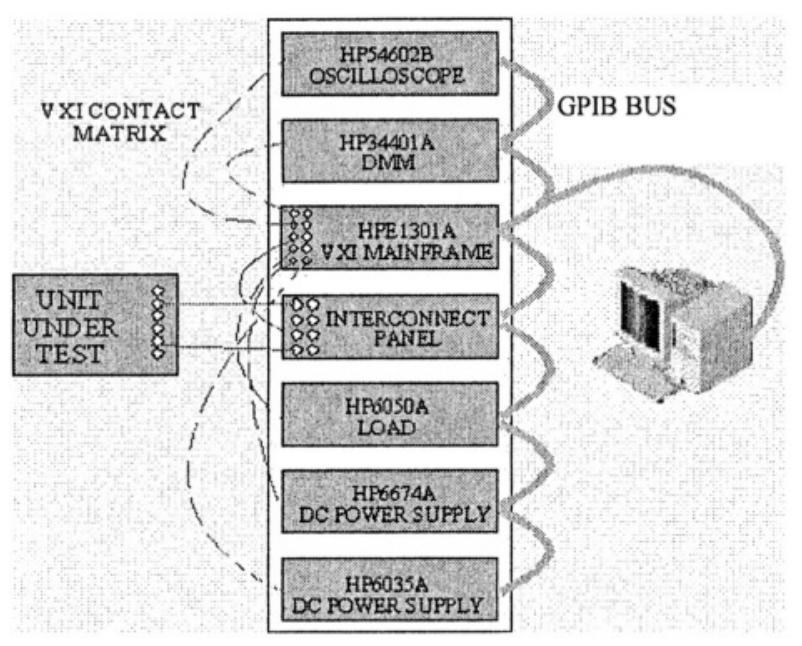

Figure 1. Block diagram RSDTC

\section{SOFTWARE DESCRIPTION}

The software developed has two main modules as shown in figure 2:

- Test Software: controls the instruments from the PC using GPIB commands.

- Network Software: communicates the host and different clients who receive information from the test bench.

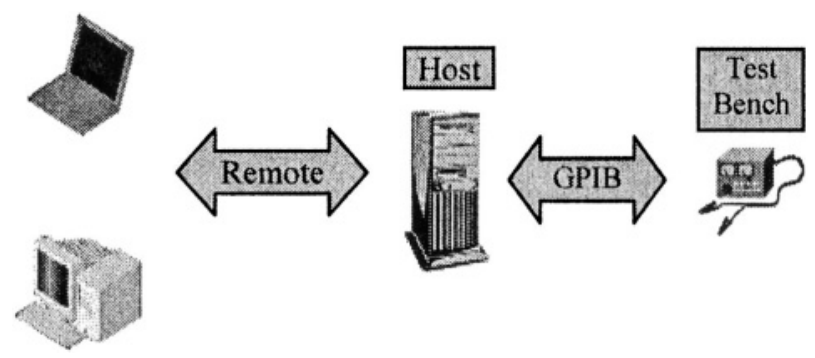

Figure 2. Software structure

\subsection{Test software}

In order to develop this module, the widely used graphical programming language LabVIEW (Laboratory Virtual Instrument Engineering Workbench), has been used. LabVIEW is a powerful and flexible 
programming language used for data acquisition, control of instruments and for analysis. [5]

The developed application is located in a personal computer that is connected to the test bench, allows the execution of tests in local and remote mode. In this last case, the PC works as a bridge between the test bench and the remote client.

Because of the instruments are controlled through a GPIB bus, the communication between the host and the instruments is based in text messages. The main part of the software is in charge to generate the necessary messages for the control of the different devices, and to understand the answer messages that are received; for it three Virtual Instruments (VI), basically related to GPIB protocol 488.2, will be used:

1. MakeAddr: generates GPIB compound addresses, necessary to control the matrix of contacts and the relays.

2. Send: it sends a text message to the element whose direction is indicated like parameter.

3. Receive: read from the GPIB bus a message of a certain length.

Following the structure of these three virtual instruments, two news virtual instrument were created: GPIBcommand and GPIBquery (show in figure 3). It's interesting to emphasize the second one, that is compounded of the Send and Receive commands. So that, in only one VI is used for request a information and then, the bus is scanned until the answer message is received. Therefore, with these two new virtual instruments, and once the addresses of the instruments included in the test bench are known, the control software automatizes the test process.

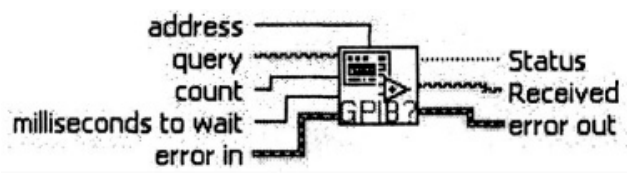

Figure 3. Virtual instrument GPIBquery

Once detailed the software that makes GPIB communication, it will be explained the two options used for the remote control of the test bench.

\subsection{Remote access application}

In this article are presented two possibilities to access to the test bench in remote way:

1. The first one is a client-server based on an application implemented by means of a communication protocol TCP/IP. 
2. The second one is based on the programming of CGI applications, so the bench can be controlled through a web browser.

Both options follow a very similar operation philosophy that is indicated in the next paragraph.

The remote access application contains two modules:

a) A program developed in LabVIEW for controlling the instruments by means of GPIB. A virtual instrument of this module must be generated with the necessary inputs and outputs for controlling and executing.

b) The second module must link the requests for information from the client to the server. That is to say, when in a client computer a button of the designed application is clicked, the effect in the GPIB communication program is the same as this one were being executed in local way.

Therefore, the differences between both communication methods are in the sending of data between the client and the server, and the process of these data to connect them to the virtual instrument control.

This communication TCP/IP has two important disadvantages:

- Is mandatory that the LabVIEW program is installed in both computers (client and server), and the developed application.

- The transfer of data is slow due to the complexity of the virtual instrument used.

For these reasons, in this work the solution based on programming CGI is only described.

\subsubsection{CGI programming}

CGI is the acronym of Common Gateway Interface. CGI is based on a communication protocol by which a Web server communicates with other applications or databases. CGI only defines an interface, is not programming language. Fig 4 shows the interface relation.

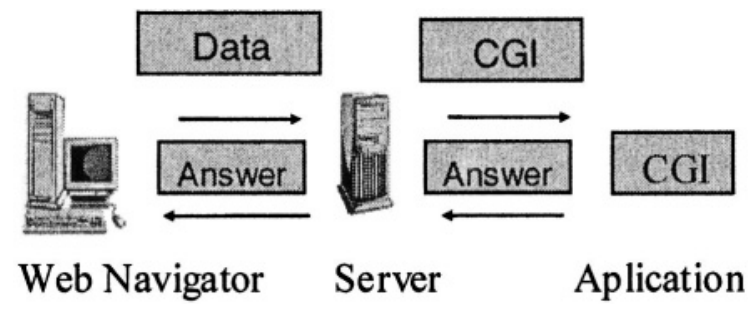

Figure 4. Interface relation

CGI applications can be written in most of the programming languages, although the most used it is the Perl language. In order to be able to use this 
communication interface in LabVIEW, the Internet tools for LabVIEW are needed. These Tools contain virtual instruments for the CGI communication [7].

When in a web browser the URL (Uniform Resource Locator) address of a CGI application is indicated, the communication process has the following steps:

- The browser sends the request to the web server. This request includes the name and the location of the CGI application, and all the input parameters of the application.

- The web server calls the CGI application, transferring all the input parameters and other available data as: the time, the client date and IP direction.

- When CGI application finalizes the execution, it gives back its output parameters to the browser.

The Internet tools of LabVIEW provide a virtual instrument called $G$ Web server, see figure 5. It is the server who uses LabVIEW to be able to connect a VI to Internet.

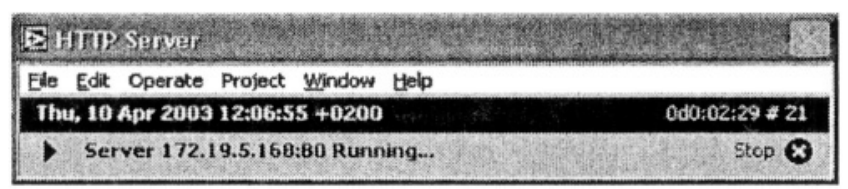

Figure 5. G Web Server

CGI application consists of three basic parts:

- A virtual instrument that makes the CGI communication between the server and the browser. Its function is to receive the request for information from the server, it sends this request to another virtual instrument that processes it, and finally, it sends the answer to the browser. The reception of this information is made by means of CGI instrument: Read Request.vi, and it is sent by CGI Release.vi.

- The virtual instrument that processes the request for information, have like main missions look for in the Keyed Array (see Table 1) with the help of the virtual instrument Keyed Array Index, the necessary inputs, and send them to the virtual instrument that is wanted to control. Finally, it is created the web page to send to the browser.

- The virtual instrument that the clients are going to be able to handle through the browser is the one in charge to communicate with the test bench. 
Table 1. Example of a Keyed Array

\begin{tabular}{cc}
\hline Key & Value \\
\hline Signature & Electronic \\
Credit & 12 \\
Course & $1^{\circ}$
\end{tabular}

The virtual instruments more important to program this CGI communication are: CGI Read Request.vi, CGI Write Reply.vi, CGI Release.vi, CGI Parse URL-Encoded Param String.vi, and Keyed Array Index.vi.

Therefore, with this software that implements CGI communication, the students can use the system, from any connected computer to Internet and through any browser, without to have installed LabVIEW.

\section{PROCEDURE FOR THE DEFINITION AND ACCOMPLISHMENT OF THE TEST}

In this section is described the procedure to follow in order to use the test bench.

At the time of defining the tests sequence, it must be distinguished between local and remote execution.

\subsection{Execution in local way}

This work mode can only be used from the computer that is connected directly with the test bench, through the GPIB card. In this mode all the configuration windows are available. The steps to follow for the definition and accomplishment of a new test are:

a) Make physical connections between UUT and the test bench.

The following steps correspond to the definitions that must be introduced in the test software.

a) Step 1. Information Editor. The new model of the UUT is named.

b) Step 2. Definition of connections. The connections between the UUT and the test bench are defined.

c) Step 3. Specifications Editor. In this section is defined the maximum and minimum voltage and currents values, that can handle the converter.

d) Step 4. Test definition. In this section the test to be realized and its sequence are defined.

Step 5. Limits and conditions test. 


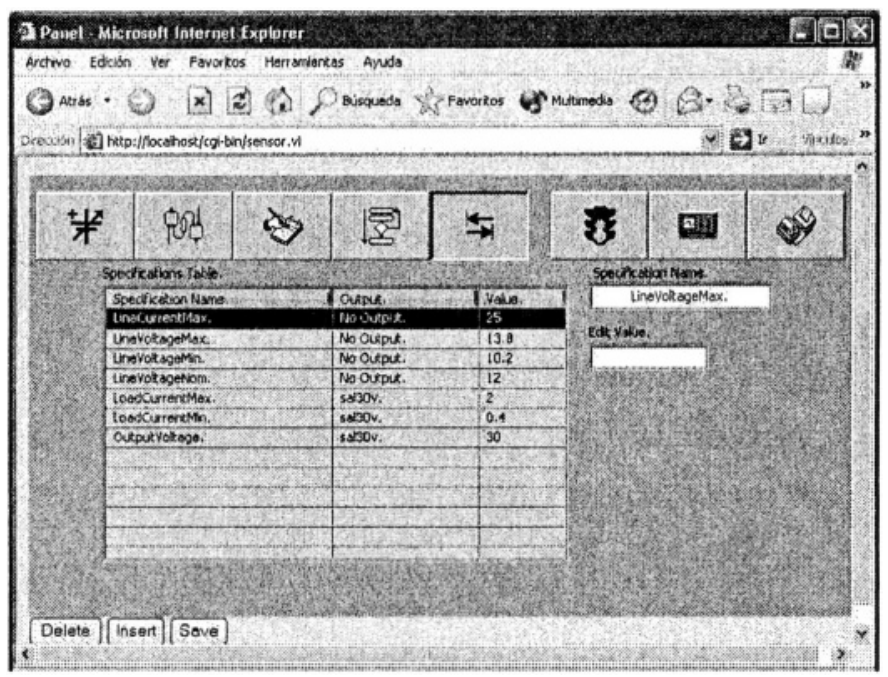

Figure 6. Remote access to the step 3 window

As an example in figure 6, is shown one of the windows in which is defined the limits values of circuit operation, obtained in remote way by means of a browser.

Once made these six steps, the system is ready for the execution of the test sequence. For it, software enables a new window in which there are a series of buttons to the tests execution, and a zone where the evolution of execution is observed. In figure 7 , is shown this window when a tests sequence has finished. 


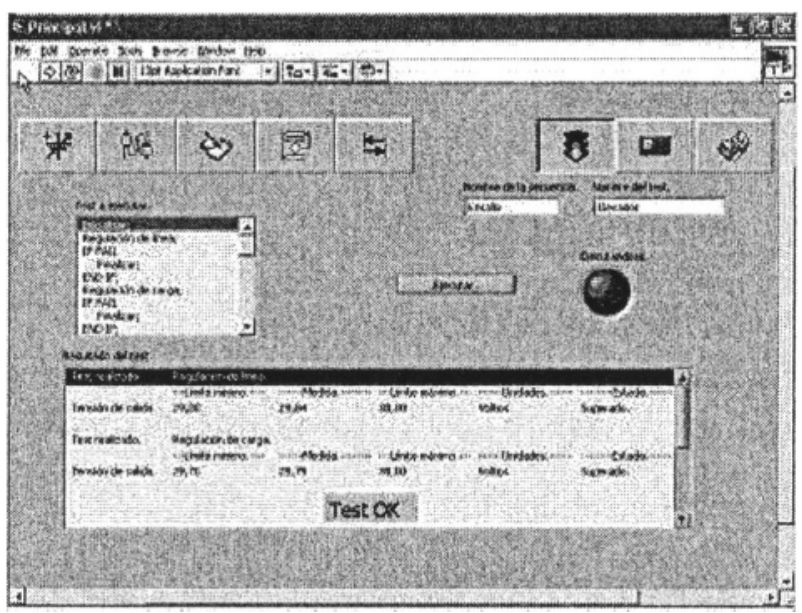

Figure 7. Test execution window

\subsection{Execution in remote mode}

If the tests are made in remote mode, the test software doesn't allow modifying the parameters as in local mode, it is only possible to visualize, the values of the three first steps. It solely allows to define a new test sequence, to introduce the tests values limits, and finally to execute the new sequence.

\section{TOPOLOGY DESIGN AND RESULTS}

As it was indicated in previous sections, the developed system is useful for the design and test of DC/DC converters.

From the design point of view, the software offers a window that allows access to all the instrumentation of the test bench. With this window, it shows in figure 8 , it is possible perform a manual configuration of the bench, choosing the connection and waveform to measure. The possibility of changing the connections allows to modify the physical structure of the converter, it facilitates the analysis of the topology.

Thus in figure 8 , it can be observed basic signals of the step-up converter: the upper waveform is the control signal to the switch, the middle represents the inductor current and the lower waveform is the output voltage. 


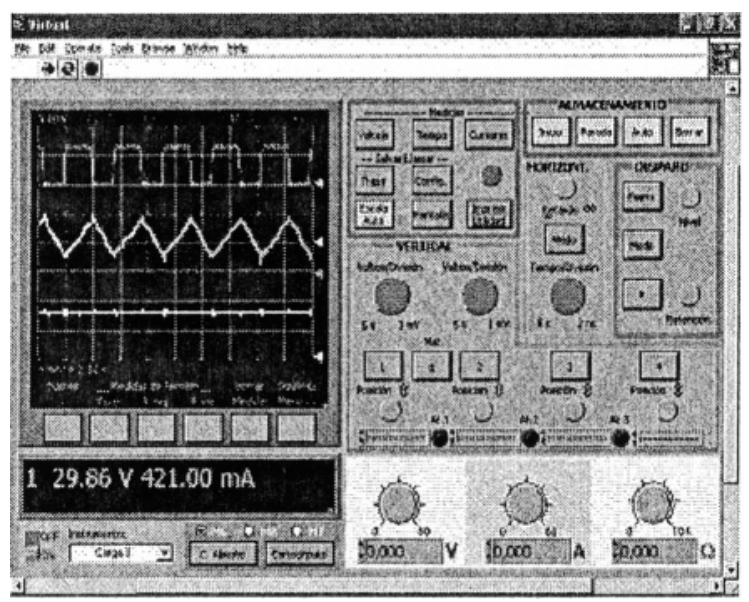

Figure 8. Circuit Operation in CCM

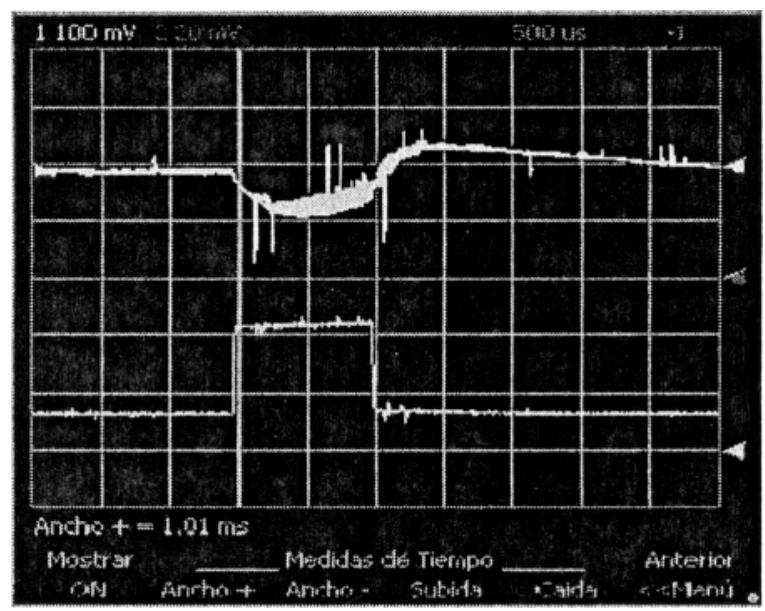

Figure 9. Result obtained with a load regulation test

In this analysis mode also is possible to visualize waveforms through the instrumentation window. As an example, in figure 18, is shown the obtained answer when executing an analysis of "current pulse in the load". The current pulse applied can be observed, that varies of 0.4 Amp. to 2 Amp. and the variation that experiences the output voltage is only of the order of $20 \mathrm{mV}$. This last measurement is solely shown the AC component, to obtain a greater definition. 


\section{CONCLUSIONS}

The tests system presented in this article can be used as a complement to the power electronics laboratory lessons, for the study and analysis of DC/DC converters. The solution that is shown here, allows to analyse the operation of this kind of circuits, obtain the basic parameters that define their answer quality: line regulation, load regulation, input impedance, output impedance, susceptibility, etc. Adding a set of switches that allow us to modify the circuit structure, it is possible to study how this changes affects the converter behaviour in: continuous and/or discontinuous operation mode. All it added to the possibility of making the tests through a browser increases the versatility and the system utility.

\section{ACKNOWLEDGMENTS}

This research work has been sponsored by Secretaría Xeral de I+D of Xunta de Galicia (Spain) in the project PGIDT01PX30304PR. This authors wish to thank the Xunta de Galicia for their financial support.

\section{REFERENCES}

[1] C. Salzmann, D. Gillet, and P. Huguenin, Introduction to real-time control using LabVIEW with an application to distance learning," Int. J. Eng. Eud. vol. 16, pp. 255272, 2000.

[2] K.K. Tan, T. H. Lee, and F. M. Leu, "Development o a distant laboratory using LabVIEW”, Int. J. Eng. Educ. vol. 16, pp. 273-282, 2000.

[3] Kok Kiong, tong Heng, and C. Y. Soh. "Internet-base monitoring of distributed control systems". IEEE Trans. Education. vol. 45 May 2002, page 128-134.

[4] ANSI/IEEE standard 488.2-1992. "IEEE standard codes, Formats, Protocols and Common Commands" The institute of Electrical an Electronics Engineers, Inc, 1988, 1993.

[5] Lisa K. Wells y Jeffrey Travis, "LabVIEW for everyone", Ed. Prentice Hall PTR.

[6] Comer, Douglas E. Y Stevens, David L. "Internetworking with TCP/IP", Ed. Prentice Hall, 2001.

[7] Jeffrey Travis, "Internet Applications in LabVIEW ”, Ed. Prentice Hall PTR. 